\title{
Halitosis en otorrinolaringología
}

\section{Halitosis in otolaryngology}

\section{Natalia Jara 01', Héctor Bahamonde $\mathrm{S}^{2}$, Juan Pablo Bravo $\mathrm{I}^{3}$.}

\section{RESUMEN}

La halitosis es una afección muy prevalente en la población mundial que genera un gran impacto en la calidad de vida de los pacientes y es un motivo frecuente de consulta en la práctica clínica diaria en otorrinolaringología, presentándonos un gran desafío para el manejo de esta condición. En general se considera que es exclusiva de la odontología, por lo que resulta interesante revisar en este artículo los factores etiológicos involucrados y los avances en diagnóstico y tratamiento.

Palabras clave: Halitosis, compuestos sulfuros volátiles, otorrinolaringología.

\begin{abstract}
Halitosis is a very prevalent disease in the world population that generates a great impact on the quality of life of patients and is a frequent complaint in daily clinical practice in otolaryngology, presenting us with a great challenge for the management of this condition. It is generally considered to be exclusive of dentistry, so it is interesting to review in this article the etiological factors involved and advances in diagnosis and treatment.
\end{abstract}

Key words: Halitosis

1 Médico Cirujano, Universidad de Chile.

2 Médico Servicio de Otorrinolaringología, Hospital Clínico Universidad de Chile.

3 Interno de Medicina, Universidad del Desarrollo. 


\section{INTRODUCCIÓN}

La halitosis o mal aliento se define como un aliento ofensivo para los otros ${ }^{1}$.

Es una condición común en la población general, se estima que afecta al $25 \%$ a $30 \%$ de la población mundial2. Genera un gran impacto no solo en la salud de los pacientes, sino que también en sus relaciones interpersonales llegando a deteriorar su calidad de vida.

Los factores involucrados son diversos incluyendo factores dietarios, hábitos, fármacos, estrés y diversas patologías. Por lo que un enfoque global de esta condición clínica es fundamental para lograr un adecuado manejo $0^{1,3,6}$. El objetivo de este artículo es revisar los avances etiológicos, diagnóstico y el manejo actual.

\section{EPIDEMIOLOGÍA}

No hay estudios epidemiológicos claros de la prevalencia de halitosis en la población mundial. Ya que la halitosis no siempre es un problema en diferentes culturas, debido a las diferencias en la apreciación de los olores. Otro factor relevante en los estudios epidemiológicos es la falta de uniformidad de criterios diagnósticos². Nadanovsky en 2007 realizó un estudio en Brasil con estudiantes universitarios y sus familias estimando que la prevalencia de halitosis es de $15 \%$. Los hombres eran 3 veces más afectados que las mujeres y la incidencia aumentaba en los mayores de 20 años de edad. Estudios en Asia muestran una prevalencia entre $6 \%$ y $23 \%$ en población general en Japón y de $20 \%$ $34 \%$ en población general China ${ }^{17}$. Lamentablemente no disponemos de cifras nacionales.

\section{FISIOPATOLOGÍA}

Fisiológicamente se produce mal olor del aire espirado al despertar. Debido a la hipo salivación nocturna, disminuyendo la síntesis de lisozima (enzima bactericida) y así provocando un aumento de la actividad metabólica bacteriana que favorecen la formación de los compuestos volátiles sulfurados (CVS) que provocan halitosis matinal, la cual tiene una breve duración y se resuelve con aseo dental, comer o beber agua fresca ${ }^{5}$.
Los CVS que más se relacionan con halitosis son el sulfuro de hidrógeno $\left(\mathrm{H}_{2} \mathrm{~S}\right)$, metilmercaptano $\left(\mathrm{CH}_{3} \mathrm{SH}\right)$ y sulfuro de dimetilo $\left(\mathrm{CH}_{3}\right)_{2} \mathrm{~S}$. Otras moléculas que participan, pero con un rol menos importante son los compuestos fenilos (indol y escatol) y las diaminas (cadaverina y putrescina). El metabolismo de las proteínas es la principal fuente de producción de compuestos volátiles. La degradación de cisteína, cistina y metionina producen compuestos de azufre volátiles. El triptófano genera diaminas, mientras que lisina y ornitina forman diaminas, en la Tabla 1 se resumen los principales compuestos volátiles involucrados en la fisiopatología de la halitosis y su composición química ${ }^{3,5,6}$.

Las patologías de cavidad oral son las causas más frecuente de halitosis. Los principales factores involucrados son una deficiente higiene oral provocando acumulación de restos alimentarios y formación de placa bacteriana, junto al uso de prótesis favorecen la degradación de restos alimentarios por bacterias anaeróbicas y Gram negativas de la cavidad oral formando compuestos volátiles sulfuros (CVS) ${ }^{5}$. Las infecciones como caries, enfermedad periodontales y la gingivitis favorecen la acumulación de bacterias, inflamación crónica y la producción de CVS $1,3,7$.

La saburra corresponde al depósito de restos alimentarios, leucocitos, bacterias y células descamativas en el dorso de la lengua, dado a su gran superficie es el principal reservorio de bacterias en la cavidad oral ${ }^{3,5}$.

Otro factor importante en la fisiopatología de la halitosis es la hiposalivación o xerostomía, la saliva es esencial para mantener la salud oral, protege a la mucosa oral, mantiene la remineralización de los dientes, ayuda en la digestión, en la sensación del sabor de los alimentos, mantiene el ph en la cavidad oral y permite una adecuada fonación. Contiene electrolitos, péptidos, glicoproteínas, lípidos, antimicrobianos y antioxidantes. En pacientes con enfermedad de Sjögren, diabetes, estrés, depresión, enfermedad tiroidea, secundaria al uso de fármacos (anticolinérgicos, antihistamínicos y diuréticos), respiradores bucales, pacientes sometidos a radioterapia y el abuso de alcohol, son situaciones que favorecen la menor producción de saliva $3,5,18$. 
Tabla 1. Moléculas volátiles relacionadas con halitosis

\begin{tabular}{|ll|}
\hline Categoría & Compuesto y composición química \\
\hline Compuestos sulfuros volátiles & Metil mercaptano: $\mathrm{CH}_{3} \mathrm{SH}$ \\
& Sulfuro de hidrogeno: $\mathrm{H}_{2} \mathrm{~S}$ \\
Diaminas & Sulfuro de dimetilo: $\left(\mathrm{CH}_{3}\right)_{2} \mathrm{~S}$ \\
& Putrescina: $\mathrm{NH}_{2}\left(\mathrm{CH}_{2}\right)_{4} \mathrm{NH}_{2}$ \\
& Cadaverina: $\mathrm{NH}_{2}\left(\mathrm{CH}_{2}\right)_{5} \mathrm{NH}_{2} \mathrm{COOH}_{2}$ \\
& Acido butírico: $\mathrm{CH}_{3} \mathrm{CH}_{2} \mathrm{CH}_{2} \mathrm{COOH}$ \\
& Acido propiónico: $\mathrm{CH}_{3} \mathrm{CH}_{2} \mathrm{COOH}$ \\
Compuestos fenilos & Acido Valérico: $\mathrm{C}_{5} \mathrm{H}_{10} \mathrm{O}_{2}$ \\
& Indol: $\mathrm{C}_{8} \mathrm{H}_{7} \mathrm{~N}$ \\
& Escatol: $\mathrm{C}_{9} \mathrm{H}_{9} \mathrm{~N}$ \\
Alcoholes & Piridina: $\mathrm{C}_{5} \mathrm{H}_{5} \mathrm{~N}$ \\
Alcalinos & 1-propoxi-2-propanol \\
Compuestos nitrogenados & 2-metil-propano \\
& Urea: $\left(\mathrm{NH}_{2}\right)_{2} \mathrm{CO}$ \\
Cetonas & Amonio: $\mathrm{NH}_{3}$ \\
\hline
\end{tabular}

Tabla adaptada de Bollen \& $\mathrm{Col}^{3}$.

\section{CAUSAS}

La halitosis es una forma de manifestación de diferentes patologías, como enfermedades sistémicas, fármacos e incluso enfermedades psiquiátricas.

Se estima que entre el $80 \%-90 \%$ de los casos está originada en la cavidad oral y que el $10 \%$ de las causas corresponden a una patología otorrinolaringológica y el $5 \%$ a otras patologías ${ }^{5-6}$.

Entre las causas otorrinolaringológicas destacan las infecciones agudas especialmente de origen bacteriano como amigdalitis, faringitis y sinusitis ${ }^{3,5}$.
La tonsilolitiasis presenta un rol importante, debido a que la superficie amigdalina alberga biofilms de bacterias y restos alimentarios, responsables del mal aliento. Se estima que representa el $3 \%$ de las causas de halitosis. Es más frecuente en adultos con una edad media de presentación de 46 años y con igual distribución por sexo. Estudios estiman que la presencia de tonsilolitos aumenta en 10 veces el riesgo de tener niveles elevados de CVS, debido a la acumulación de detritus bacterianos $^{3,10}$. En la Tabla 2 se presentan las principales enfermedades otorrinológicas relacionadas con halitosis y las bacterias responsables de producción de CVS.

Tabla 2. Enfermedades otorrinológicas relacionadas con halitosis y el microorganismo productores de CVS $^{3}$

\begin{tabular}{|ll|}
\hline Patología & Microorganismo relacionado con halitosis \\
\hline Amigdalitis aguda & Estreptococo \\
Mononucleosis & Virus Epstein Barr \\
Angina de Plaut-Vincent & Fusobacterium, Bacteroides \\
& Borrelia, Treponema \\
Tonsilolitos & Eubacterium, Fusobacterium, \\
& Porphyromonas, \\
Sinusitis & Prevotella, Selenomonas, Tanerella \\
& Estreptococo pneumonia, Haemophilus \\
& influenza \\
\hline
\end{tabular}


Es importante considerar como causa de halitosis en los niños la presencia de cuerpos extraños en la cavidad nasal, malformaciones congénitas como el paladar hendido y labio leporino. En adultos debemos considerar procesos inflamatorios crónicos como la rinitis atrófica y condiciones iatrogénicas como el síndrome de nariz vacía ${ }^{3,11}$.

Patologías pulmonares como bronquiectasias, abscesos pulmonares, enfermedad pulmonar obstructiva crónica, neoplasias pueden provocar halitosis ${ }^{3}$.

Se ha descrito en la literatura que infecciones por pseudomonas auriginosa favorecen la síntesis de aminoacetofeno responsable de mal aliento ${ }^{5}$.

Las enfermedades gastrointestinales son una causa infrecuente de halitosis. El aumento de infección por Helicobacter pylori, en especial en países pobres, con deficientes medidas sanitarias ha significado una mayor frecuencia. En Sudamérica la tasa de prevalencia de infección por Helicobacter pylori es de $24,3 \%$ a $61 \%^{12}$. Este microorganismo es el principal factor patogénico de inflamación y cambios ulcerativos en la mucosa gástrica su presencia se asocia a úlceras gastroduodenales, reflujo gastroesofágico y cáncer gástric $0^{13}$. Lee en 2006 demostró que Helicobacter pylori es capaz de producir sulfuro de hidrógeno y metilmercaptano, responsables del mal aliento. Diversos estudios han demostrado que la erradicación de Helicobacter pylori ha logrado mejorar la halitosis ${ }^{16}$.

Las alteraciones metabólicas de origen genético 0 adquirido en escasas oportunidades pueden generar CVS, especialmente el sulfuro de dimetilo, el cual es transportado por el sistema circulatorio y excretado por el pulmón, alterando el aire exhalado. Entre estas condiciones destacan la descompensación de la diabetes mellitus, insuficiencia renal crónica, insuficiencia hepática, trimetilaminuria, cistinosis y la hipermetioninemia ${ }^{5}$.

La halitosis puede ser consecuencia de malos estilos de vida como el consumo de café, tabaco, alcohol, bebidas y alimentos odoríferos. Un análisis del humo del tabaco demostró que contiene compuestos de azufre volátiles detectables en mediciones con Halimeter ${ }^{17,21}$, estos compuestos alteran la estructura y función de la saliva. Sumado a que los pacientes fumadores tienen una menor tasa de producción de saliva, lo que favorece la halitosis, las enfermedades periodontales y pérdidas dentales ${ }^{18}$. Algunos alimentos con sustancias odoríferas como el ajo, cebolla, especies, repollo, coliflor y rábanos son causa común de halitosis ${ }^{19}$. El consumo de alcohol ha demostrado objetivamente que aumenta la producción de CVS y alteración del test organoléptico ${ }^{20}$.

Es importante tener presente factores psicológicos y psiquiátricos al momento de enfrentar casos de halitosis, en especial cuando el diagnóstico del paciente ha sido difícil de objetivar o en casos de fracaso del tratamiento. En estas circunstancias debemos considerar la hálito-fobia que es el temor a tener mal aliento, es un tipo de fobia social y se estima que el $0,5 \%$ a $1 \%$ de la población adulta presenta esta alteración ${ }^{3}$. Otra patología psiquiátrica es la pseudo-halitosis, que corresponde a mal olor del aire expirado referido por el paciente que no puede ser objetivado por el equipo de salud, este cuadro clínico se presenta con mayor frecuencia en pacientes con depresión ${ }^{14}$. Estudios muestran que estados de ansiedad y personalidades con rasgos neuróticos aumentan la producción de CVS y trimetilamina ${ }^{22}$. El tratamiento de elección en estos casos es un manejo multidisciplinario con psiquiatría y psicoterapia ${ }^{3,5}$.

\section{DIAGNÓSTICO}

En vista de la amplia gama de causas que pueden provocar halitosis resulta fundamental realizar una anamnesis detallada en los antecedentes mórbidos y familiares, enfatizando en los hábitos y la dieta. El examen físico detallado en la cavidad oral y la búsqueda de signos de enfermedades sistémicas son relevantes para llegar a un correcto diagnóstico.

Los métodos diagnósticos que han sido utilizados son la medición organoléptica y medición de compuestos de azufre volátiles por medio de dispositivos electrónicos que revisaremos a continuación.

La medición organoléptica corresponde al Gold standard actual para el diagnóstico de halitosis, ya que permite cuantificar más de 150 componentes del aire espirado. El test cuando es realizado por tres jueces entrenados alcanza una correlación de más $83 \%^{8}$. Las ventajas de este examen es su bajo costo, no requiere equipos costosos, detecta una amplia gama de olores, se puede realizar en la práctica 
clínica diaria. Las desventajas son la subjetividad, falta de cuantificación, saturación de la percepción olfatoria del examinador y la reproducibilidad del test $^{3,5,8}$. El test se realiza por tres jueces diferentes en 20 más ocasiones diferentes, se evalúa el aire espirado del paciente por medio de un tubo, en un extremo el paciente respira normalmente y en el otro extremo el examinador evalúa el aire espirado, también se analiza la saburra, se raspa el dorso de la lengua y se analiza. Se otorga una puntuación según intensidad del olor, que va de 0 a 5 puntos ${ }^{3}$. La escala de evaluación junto con las recomendaciones para realizar el test se describe en la Tabla 3.

La cromatografía de gases puede determinar la concentración de los CVS en el aliento, saliva y saburra. Detecta casi 500 sustancias diferentes, como alcoholes, compuestos fenilos, alcanos, cetonas y compuestos nitrogenados ${ }^{8}$. Dentro de sus ventajas incluye análisis de casi todos los componentes del aire espirado con una alta sensibilidad y especificidad, es un examen reproducible y objetivo. No es un examen invasivo. Sus desventajas que es un examen costoso, requiere de personal capacitado en el uso del equipo y requiere de mucho tiempo para realizar la medición por estas razones no se utilizan de rutina en la práctica clínica ${ }^{3}$.

Entre los dispositivos electrónicos portátiles que detectan CVS del aire espirado está Halimeter, que nos proporciona una idea general de la cantidad de CVS pero no permite diferenciar entre los principales CVS, su ventaja que es portátil, fácil de utilizar, rapidez de los resultados y reproducibilidad de ellos ${ }^{5,23}$.
Oral Chroma ${ }^{\mathrm{TM}}$ es un dispositivo portátil fácil de utilizar y de bajo costo permite cuantificar los tres principales CVS relacionados con la halitosis (sulfuro de hidrógeno, metilmercaptano, sulfuro de dimetilo) ${ }^{3,23}$. Actualmente los valores de referencias para realizar el diagnóstico de halitosis están en periodo de validación y estandarización, los fabricantes de Oral Chroma $^{\mathrm{TM}}$ definen halitosis con niveles de $\mathrm{H}_{2} \mathrm{~S}$ mayor 112 ppb y más de 26 ppb para $\mathrm{CH}_{3} \mathrm{SH}$. Un estudio en pacientes sanos determinó niveles matinales de CVS de H2S: $95 \mathrm{ppb}^{*}, \mathrm{CH}_{3} \mathrm{SH}: 12 \mathrm{ppb}$ y $\left(\mathrm{CH}_{3}\right)_{2} \mathrm{~S}: 24 \mathrm{ppb}^{23}$. Para Halimeter los niveles normales son menores a 100 ppb, para realizar diagnóstico de halitosis se consideran niveles sobre 300-400 $\mathrm{ppb}^{3}$.

\section{TRATAMIENTO}

El tratamiento de la halitosis está determinado por la causa, en general el manejo de las causas orales está dividido en cuatro pilares.

\section{Reducción mecánica de microorganismos y nutrientes en la cavidad oral}

Los restos alimentarios entre las piezas dentales junto con la concentración de bacterias en el dorso de la lengua son los principales factores de la halitosis. La higiene de la cavidad oral debe ser regular y se recomienda el uso de cepillo dental con pasta, aseo del dorso de la lengua, uso de seda dental después de cada comida ${ }^{1,3,5,7}$.

Tabla 3. Test de medición organoléptica²1

\begin{tabular}{|cl|}
\hline Escala de Rosemberg \& Mc Culloch & Descripción \\
\hline 0 & Sin olor detectable \\
1 & Olor casi detectable \\
2 & Olor suave \\
3 & Olor moderado \\
4 & Olor fuerte \\
5 & Olor extremadamente fuerte \\
\hline
\end{tabular}

Nota: Instrucciones antes de realizar el examen

- 24 horas antes no debe consumir alimentos odoríferos y tabaco.

- 12 horas antes del examen no se debe lavar los dientes, enjuagar o utilizar productos perfumados.

- 6 horas antes evitar la ingesta de alimentos o líquidos.

* ppb: Partes por millón. 
En una revisión sistemática en 2010 se demostró que una limpieza mecánica del dorso de la lengua reduce la saburra y el mal aliento ${ }^{24}$. Una revisión sistemática de Cochrane que comparó el aseo del dorso de la lengua con lavado de dientes y uso de productos de limpieza para reducir la halitosis en adultos demostró que el aseo del dorso de la lengua disminuye significativamente los niveles de compuestos volátiles sulfurados del aire espirado en comparación a los otros métodos analizados ${ }^{25}$.

\section{Reducción química de microorganismos}

Agentes químicos en pastas dentales y enjuagues bucales proporcionan un efecto antimicrobiano ${ }^{1}$. Las pastas de dientes que contengan fluoruro de estaño, zinc y especialmente triclosán podrían reducir el mal aliento hasta 12 horas después de cepillarse los dientes ${ }^{3,26}$. No hay estudios que demuestren un efecto real del uso y componentes de las pastas dentales en el manejo de la halitosis ${ }^{3}$.

En relación a los enjuagues bucales son frecuentemente utilizados en el tratamiento de la halitosis. En general se recomienda que se utilicen 2 ó 3 veces al día por 30 segundos, los enjuagues que contienen gluconato de clorhexidina $(\mathrm{CHX})$ en bajas dosis, cloruro de cetilpiridinio (CPC) 0 triclosán son los únicos que han mostrado un beneficio real en disminuir el mal aliento a corto plazo ${ }^{1,5}$. Por ejemplo, clorhexidina demostró que en una concentración al 0,2\% reduce en $43 \%$ los CVS con un efecto de 24 horas y mejora en $50 \%$ la puntuación en el test organoléptico ${ }^{3}$.

El triclosán es un eficaz bactericida de las principales bacterias orales, logra una reducción del $84 \%$ de los CVS después de 3 horas de aplicación ${ }^{1,3}$. El peróxido de hidrógeno a una concentración de $3 \%$ logra reducir $90 \%$ los CVS después de 8 horas de aplicación ${ }^{3}$.

\section{Conversión de CVS fragantes a compuestos no volátiles}

Estudios han demostrado que a los enjuagues bucales cuando se les agregan iones metálicos y/0 agentes oxidantes en bajas concentraciones, como el peróxido de hidrógeno, dióxido de cloro, cloruro de iminio y cloruro de zinc tiene un efecto neutralizante de los CVS y disminuyen su producción $n^{3,5}$. Un ejemplo es el enjuague bucal Halita ${ }^{\circledR}$ que contiene cloruro de cetilpiridinio, clorhexidina y lactato de zinc.

\section{Enmascaramiento del mal olor}

Productos cosméticos como gomas de mascar, tabletas, aerosoles bucales, enjuagues bucales pueden reducir en corto plazo el mal aliento y también favorecen la producción de saliva ${ }^{1,3}$.

En casos de xerostomía se recomienda el uso de captosol es un fosfato de calcio sobresaturado que se utilizan como agente oral tópico en los enjuagues bucales. Útil para el tratamiento de la serositis e hiposalivación en cavidad oral y garganta, en especial es pacientes en tratamientos de quimio y radioterapia. Este agente es capaz de aumentar la secreción de saliva. Aún faltan estudios que prueben su efectividad en la reducción de la halitosis ${ }^{8}$.

El uso de antibióticos es una alternativa infrecuente y sólo se reserva para casos refractarios a tratamiento y recomienda el uso de metronidazol en bajas dosis por una semana con el objetivo de reducir las concentraciones bacterianas anaeróbicas en la cavidad bucal ${ }^{5}$.

\section{TRATAMIENTO DE LA HALITOSIS EN OTORRINOLARINGOLOGÍA}

En general las causas más frecuentes de halitosis en la especialidad son los procesos infecciosos, cuyo manejo es con antibióticos por un periodo determinado.

En casos seleccionados de tonsilolitiasis se recomienda realizar una amigdalectomía, las técnicas empleadas pueden ser una amigdalectomía total clásica 0 una resección parcial con láser ${ }^{10}$.

En presencia de cuerpos extraños en la cavidad nasal y malformaciones congénitas del paladar como causas de halitosis, el manejo generalmente es quirúrgico $0^{3,5}$.

En pacientes con inflamaciones crónicas de la mucosa nasal como en rinitis atrófica y síndrome de nariz vacía, el uso de soluciones hidratantes, aseo nasal con soluciones salinas y corticoides locales resultan beneficiosos, en escasas situaciones el tratamiento es la reconstrucción de cornetes por medio de implantes para reducir el volumen de la cavidad nasal27,28. 


\section{TRATAMIENTO HALITOSIS DE CAUSA EXTRA ORAL}

Es fundamental para el tratamiento la causa subyacente por lo que el paciente debe ser evaluado por especialistas y con un manejo multidisciplinario. En caso de pacientes con pseudohalitosis o halitofobia la sospecha clínica y experiencia del clínico es fundamental para llegar al diagnóstico y realizar un manejo en conjunto con el equipo de salud mental.

Es fundamental el cambio de hábito evitando fumar, ingesta de alcohol y alimentos con sustancias odoríferas que contribuyan con la halitosis. La ingesta de comida en horarios establecidos y el consumo frecuente de frutas o vegetales con fibra, en especial al final de las comidas podrías ser beneficioso para reducir el mal aliento $0^{5,7}$.

\section{CONCLUSIONES}

La halitosis tiene una gran prevalencia a nivel mundial y es un motivo frecuente de consulta.

Para realizar un correcto manejo de estos pacientes debemos tener presente que es un síntoma de alguna enfermedad y que contribuyen diversos factores como hábitos, factores orales y extra orales.

Es importante destacar que no hay un criterio objetivo para realizar el diagnóstico de halitosis ni contamos con exámenes de laboratorios de fácil acceso para lograr un adecuado diagnóstico.

Un método útil serían los dispositivos electrónicos portátiles pero actualmente están en periodo de estandarización de los niveles para poder realizar el diagnóstico de halitosis y el costo económico limita su uso habitual en la práctica clínica ${ }^{3,23}$.

En relación al tratamiento de la halitosis es fundamental la educación y cambio de hábitos en los pacientes. El aseo del dorso de la lengua es la medida que mejor efecto tiene en el manejo de la halitosis ${ }^{24}$, los enjuagues bucales que contienen clorhexidina en bajas dosis, cetilpiridinio, triclosán o zinc, son los únicos que han demostrado tener un efecto beneficioso como bactericidas y disminuyendo la concentración de CVS, mejorando la halitosis 5 .

Estilos de vida saludables junto con hábitos de higiene bucal es, sin duda, la mejor estrategia de prevención y en ocasiones tratamiento para algunos tipos de halitosis 5 .

Debemos considerar casos en que no hay una causa específica de halitosis y necesitamos realizar un manejo multidisciplinario con odontólogos, otorrinolaringólogos, médicos internistas e incluso psiquiatras.

\section{BIBLIOGRAFÍA}

1. Rösing CK, LoEsche W. Halitosis: an overview of epidemiology, etiology and clinical management. Brazilian Oral Research 2011; 25 (5): 466-71.

2. Zalewska A, Zatonski M, Jablonka-Strom $A$, Paradowska A, Kawala B, Litwin A. Halitosis: a common medical and social promblem. A review on pathology, diagnosis and treatment. Acta Gastro-enterologica Belgica 2012; 75 (3): 300-9.

3. Bollen C, BeIKLeR T. Halitosis: the multidisciplinary approach. International Journal of Oral Science 2012; 4: 55-63.

4. Nadanovsky P, Carvalho LB, Ponce de leon A. Oral malodour and its association with age and sex in a general population in Brazil. Oral Diseases 2007; 13 (1): 105-9.

5. Scully C, Greenman J. Halitology (breath odour: aetiopathogenesis and Management). Oral Diseases 2012; 18: 333-45.

6. Porter SR. Diet and halitosis. Current Opinion in Clinical Nutrition and Metabolic Care 2011; 14 : 463-8.

7. Ademovski SE, Lingström P, Winkel E, Tangerman A, Persson G R, Renvert S. Comparison of different treatment modalities for oral halitosis. Acta Odontologica Scandinavica 2012; 70: 224-33.

8. KIZHNER V, Xu D, KRESPI YP. A new tool mesuring oral malador quality of life. Eur Arch Otorhinolaryngol 2011; 268: 1227-32.

9. Kinberg S, Stein M, Zion N, Shaoul R. The gastrointestinal aspects of halitosis. Can $J$ Gstroenterol 2010; 24(9): 552-6.

10. Siber S, Hat J, Brakus I, Biocic J, Brajdic D, Zajc I, Bosan- Kilibarda I, Macan D. Tonsillolithiasis and orofacial pain. Gerodontology 2012; 29: el 157-el 160.

11. Chhabra N, Houser S. The diagnosis and management of empty nose syndrome. 
Otolaryngologic Clinics of North America 2009; 42: 311-30.

12. Goh K-L, Chan W-K, ShiotaS, Yamaoka Y. Epidemiology of Helicobacter pylori infection and public health implications. Helicobacter 2011; 16 (1): 1-9.

13. Dore M, Fanciulli G, Tomasi P, Realdi G, Delitala G, Graham D, Malaty H. Gastrointestinal Symptoms and Helicobacter pylori infection in school-age children residing in porto torres, sardinia, Italy. Helicobacter 2012; 17: 369-73.

14. Suzuki N, Yoneda M, Naito T et al. Association between oral malodour and psychological characteristics in subjects with neurotic tendencies complaining of halitosis. International Dental Journal 2011; 61(2): 57-62.

15. Lee H, Kho HS, Chung JW, Chung SC, Kim YK. Volatile sulfur compounds produced by Helicobacter pylori. Journal of Clinical Gastroenterology 2006; 40 (5): 421-6.

16. Tiomny E, Arber N, Moshrowitz M, Peled Y, Gilat T. Halitosis and Helicobacter Pylori. A possible link? Journal of Clinical Gastroenterology 1992; 15: 236-7.

17. Bornstein M, Kislig K, Hoti B, Seemann R, Lussi A. Prevalence of halitosis in the population of the city of Bern, Switzerland: A study comparing self-reported and clinical data. European Journal of Oral Sciences 2009; 117: 261-7.

18. Rad M, Kakoie S, BrojenI F, Pourdamghan N. Effect of long-term smoking on whole-mouth salivary flow Rte and oral health. Journal of Dental Research, Dental Clinics, Dental Prospects 2010; 4(4): 110-4.

19. SuÁrez F, Springfield J, Furne J, Levitt M. Differentiation of mouth versus gut as site of origin of odoriferous breath gases after garlic ingestion. The American Journal of Physiology 1999; 276: G425-G430.
20. Suzuki N, Yoneda M, Naito T, Imamoto T, Yamada K, Hisama K, OKada I, HiRofuJI T. The relationship between alcohol consumption and oral malodour. International Dental Journal 2009; 59(1): 31-4.

21. Rosenberg M. Clinical assessment of bad breath: current concepts. Journal of The American Dental Association 1996; 127: 475-82.

22. YAEGAKI K, CoIL JM. Examination, classification, and treatment of halitosis; clinical perspectives. Journal Canadian Dental Association 2000; 5: 257-61.

23. Snel J, Burgering M, Smit B, Noordman W, Tangerman A, Winkel E, Kleerebezem M. Volatile sulphur compounds in morning breath of human volunteers. Archives of Oral Biology 2011; 56: 29-34.

24. Van der Sleen Mi, Slot DE, van Trijffel E et al. Effectiveness of mechanical tongue cleaning on breath odour and tongue coating: a systematic review. International Journal of Dental Hygiene 2010; 8(4): 258-68.

25. Outhouse TL, Fedorowicz Z, Keenan JV, AlAlawI R. A Cochrane systematic review finds tongue scrapers have short term efficacy in controlling halitosis. General Dentistry 2006; 54 (5): 352-9.

26. Sharma NC, Galustians HJ, Qaolsh J et al. Clinical effectiveness of a dentifrice containing triclosan and a copolymer for controlling breath odor. American Journal of Dentistry 2007; 20 (2): 79-82.

27. HOUSER S. Surgical treatment for empty nose syndrome. Arch Otolaryngology Head Neck Surg 2007; 133: 858-63.

28. Hildendrand $T$, Weber $R$, Brehmer D. Rhinitis sicca, dry nose and rhinitis: a review of the literature. Eur Arch Otorhinolaryngol 2011; 268: 17-26.

Dirección: Héctor Bahamonde S. 